\title{
Criminologie
}

\section{Pratiques de déjudiciarisation de la maladie mentale : le modèle de l'Urgence psychosociale-justice}

\section{Daphné Morin, Pierre Landreville et Danielle Laberge}

Volume 33, numéro 2, automne 2000

Problèmes sociaux et système pénal

URI : https://id.erudit.org/iderudit/004727ar

DOI : https://doi.org/10.7202/004727ar

Aller au sommaire du numéro

\section{Éditeur(s)}

Les Presses de l'Université de Montréal

ISSN

0316-0041 (imprimé)

1492-1367 (numérique)

Découvrir la revue

Citer cet article

Morin, D., Landreville, P. \& Laberge, D. (2000). Pratiques de déjudiciarisation de la maladie mentale : le modèle de l'Urgence psychosociale-justice.

Criminologie, 33(2), 81-107. https://doi.org/10.7202/004727ar
Résumé de l'article

Au Canada, on a constaté un nombre grandissant de personnes ayant des problèmes de santé mentale et qui ont des démêlés avec la justice. La dynamique d'exclusion engendrée par cette intervention pour ces personnes difficultés d'accéder à des soins et des services, détérioration des conditions de vie, accélération de la judiciarisation avec des retours multiples en prison -, ainsi que l'épuisement des ressources - consultations répétées en urgence psychiatrique, demandes accrues de services auprès des ressources communautaires -, ont montré la nécessité d'examiner la politique de déjudiciarisation. Cette dernière vise l'usage de mesures non pénales ou, lorsque ce n'est pas possible, la diminution ou l'absence de recours à l'incarcération, selon la nature ou la gravité du problème à régler. Cet article documente des pratiques de déjudiciarisation à travers l'exploration d'un nouveau programme d'intervention : l'Urgence psychosociale-justice de Montréal (UPSJ). Après un examen de la thèse de la criminalisation de la maladie mentale, le texte présente quelques résultats préliminaires des 19 premiers mois de fonctionnement du service. En conclusion, s'il apparaît évident que l'UPS joue un rôle clé en amont en évitant des entrées dans le système pénal, elle soulève la question de la capacité du milieu à répondre aux besoins d'intervention et de prise en charge des populations ciblées. 


\section{Pratiques de déjudiciarisation de la maladie mentale : le modèle de l'Urgence psychosociale-justice}

\author{
Danielle Laberge \\ professeure \\ Département de sociologie \\ Université du Québec à Montréal \\ laberge.danielle@uqam.ca \\ Pierre Landreville \\ professeur \\ École de criminologie \\ Université de Montréal \\ pierre.landreville@umontreal.ca
}

\author{
Daphné Morin \\ professionnelle de recherche \\ collectif de recherche \\ sur l'itinérance, la pauvreté et l'exclusion sociale \\ Université du Québec à Montréal \\ morin.daphne@uqam.ca
}

RÉSUmé - Au Canada, on a constaté un nombre grandissant de personnes ayant des problèmes de santé mentale et qui ont des démêlés avec la justice. La dynamique d'exclusion engendrée par cette intervention pour ces personnes - difficultés d'accéder à des soins et des services, détérioration des conditions de vie, accélération de la judiciarisation avec des retours multiples en prison - , ainsi que l'épuisement des ressources - consultations répétées en urgence psychiatrique, demandes accrues de services auprès des ressources communautaires - , ont montré la nécessité d'examiner la politique de déjudiciarisation. Cette dernière vise l'usage de mesures non pénales ou, lorsque ce n'est pas possible, la diminution ou l'absence de recours à l'incarcération, selon la nature ou la gravité du problème à régler. Cet article documente des pratiques de déjudiciarisation à travers l'exploration d'un nouveau programme d'intervention : l'Urgence psychosociale-justice de Montréal (UPSJ). Après un examen de la thèse de la criminalisation de la maladie mentale, le texte présente quelques résultats préliminaires des 19 premiers mois de fonctionnement du service. En conclusion, s'il apparaît évident que l'UPSJ joue un rôle clé en amont en évitant des entrées dans le système pénal, elle soulève la question de la capacité du milieu à répondre aux besoins d'intervention et de prise en charge des populations ciblées. 
ABSTRACT - A growing numbers of people who suffer from mental health problems have been found to run into trouble with the criminal justice system. The exclusion dynamic created by intervention targeted at these people - difficult medical care and access to services, deteriorating living conditions, the acceleration of judicial processing along with multiple prison experiences - as well as increasing ressource limitations repeated psychiatric emergency ward consultations, growing service demands on community ressources - have both demonstrated the need to examine policies that would divert people from the criminal judicial system. Such policies aim to promote the use of non judicial measures, or when this is not possible, lessen or put an end to incarceration practices, depending on the nature and seriousness of the problem. This article researches judicial diversion practices through the exploration of a new intervention program : l'Urgence psychosociale-justice de Montréal (UPSJ). After reviewing the mental illness criminalization thesis, the text goes on to present preliminary results of the service's first 19 months of functioning. In conclusion, although the UPSJ obviously plays a key role in reducing entries into the criminal justice system, its very existence also raises the issue of the environment's capacity to answer the target population's intervention and caretaking needs.

Depuis déjà de nombreuses années, le développement des politiques au Québec et au Canada s'est orienté vers un usage parcimonieux de la judiciarisation (CRDC, 1975; 1976). En effet, les coûts humains et financiers du recours au système de justice, et particulièrement de justice pénale, sont importants et peuvent être excessifs par rapport aux résultats escomptés. Cette orientation générale des politiques ne signifie certainement pas, comme certains le craignent, une tolérance de l'inacceptable, une déresponsabilisation des individus. Il s'agit plutôt de trouver des solutions qui minimisent le recours au système pénal et diminuent les coûts sociaux et personnels d'une telle intervention. Le nombre grandissant de personnes ayant des problèmes de santé mentale et qui ont des démêlés avec la justice a montré la nécessité d'examiner ces politiques dans une perspective large et dépassant les seules questions juridiques. Les préoccupations se sont centrées sur la dynamique d'exclusion et de marginalisation engendrée par l'intervention de la justice pour ces personnes : difficultés d'accéder à des soins et des services, détérioration des conditions de vie, accélération de la judiciarisation avec des retours multiples en prison, consultations répétées en urgence psychiatrique, épuisement des ressources communautaires. L'incapacité structurelle d'intervenir rapidement et de coordonner les interventions entre les différentes instances impliquées viendrait alimenter cette dynamique. Les différents acteurs concernés en sont arrivés à un consensus qui favorise la déjudiciarisation. Celle-ci vise 
l'usage de mesures non pénales ou, lorsque ce n'est pas possible, la diminution ou l'absence de recours à l'incarcération, selon la nature ou la gravité du problème à régler. Une telle approche ne se limite pas à la seule diminution de l'usage du système pénal, mais reconnaît aussi la nécessité de trouver des solutions aux problèmes vécus par la personne elle-même ou par son entourage.

L'Urgence psychosociale-justice de Montréal (UPSJ) est un des moyens développés dans le cadre de la mise en œuvre de politiques de déjudiciarisation ${ }^{1}$. Elle constitue l'aboutissement de plusieurs années de débats, de recherches et de concertation autour de cette problématique au Québec et, en particulier, à Montréal. Il s'agit d'une ressource d'intervention d'urgence, offrant des services 24 heures sur 24 et 7 jours par semaine, mise sur pied en octobre 1996, et qui vise explicitement la déjudiciarisation des personnes ayant des problèmes de santé mentale et ceci, à travers un modèle novateur. L'UPSJ rejoint non seulement les individus qui sont la cible des interventions "psychiatrie-justice », mais également les ressources ou services qui sont appelés à jouer un rôle dans les pratiques de « diversion » du système pénal et de réinsertion sociale. On pense en particulier à la police, aux tribunaux, aux urgences, aux hôpitaux et cliniques externes en psychiatrie, aux centres de crise, aux ressources communautaires qui œuvrent dans le domaine de l'hébergement, du support et du suivi dans la communauté (centres de jour, suivi communautaire intensif, etc.). Bref, il s'agit d'un organisme dont le mandat vise à favoriser la concertation entre ces diverses agences afin d'atteindre ses objectifs de déjudiciarisation et de réinsertion. Le mode de financement du service rend également compte de ce rôle d'interface assigné au service. En effet, si la part principale du financement provient de la Régie régionale de la santé et des services sociaux de MontréalCentre (ministère de la Santé et des Services sociaux), d'autres contributions, sous forme monétaire ou de prêt de service, sont fournies par d'autres sources dont le ministère de la Sécurité publique et l'Institut Philippe Pinel de Montréal.

1. Différents modèles de ce type ont été mis sur pied aux États-Unis, en GrandeBretagne et en Nouvelle-Zélande au cours des années 1980 et au début des années 1990. Ces programmes présentent de nombreuses variantes mais, dans la majorité des cas, ils se sont développés pour fournir une alternative à la criminalisation de la maladie mentale (Finn et Sullivan, 1989; Cohen et Tsemberis, 1991; Fennell, 1991; James et Hamilton, 1991, 1992; Joseph et Potter, 1993a,b; Brinded et al., 1996). 
Cet article vise à documenter des pratiques de déjudiciarisation de la maladie mentale au Québec à travers l'exploration de ce nouveau programme d'intervention. La première partie examine la thèse de la criminalisation de la maladie mentale avec les prémisses qu'elle soustend et les conséquences qu'une telle option engendre. La deuxième partie situe le contexte d'émergence de l'UPSJ à Montréal et présente quelques résultats préliminaires des 19 premiers mois de fonctionnement du service.

\section{Maladie mentale et système pénal}

\section{La criminalisation de la maladie mentale}

Depuis près de 30 ans, de nombreuses études nord-américaines se sont penchées sur le phénomène de la «criminalisation de la maladie mentale » (Abramson, 1972; Bonovitz et Bonovitz, 1981; Teplin, 1983; Davis, 1992; Wachholz et Mullaly, 1993; Brinded et al., 1995; Green, 1997; Patch et Arrigo, 1999). Cette expression, utilisée la première fois par le chercheur américain Abramson, désignait essentiellement le phénomène de prise en charge, par les instances du système de justice criminelle, de personnes dont la caractéristique principale était de souffrir de problèmes de santé mentale. Les préoccupations d'Abramson, tout comme celles d'autres chercheurs par la suite, s'inscrivaient dans le cadre d'importantes transformations dans les conceptions de la maladie mentale et dans les modèles de dispensation des soins de santé mentale (Foucault, 1961; Scheff, 1966; Goffman, 1968; Castel, 1981; Warren, 1981). Ces transformations, sur le plan des pratiques hospitalières et des représentations du traitement adéquat, avaient considérablement affecté les lieux de vie d'un nombre important de personnes qui, jusque-là, avaient été prises en charge, partiellement ou complètement, par les hôpitaux psychiatriques.

À la même époque, on assistait, à travers l'Amérique du Nord, à des modifications législatives concernant les critères d'internement civil involontaire. Ces modifications, malgré certaines variations locales, étaient toutes orientées dans la direction d'un accroissement des conditions nécessaires pour procéder à l'internement d'une personne contre son gré (Gordon et Verdun-Jones, 1986; Robertson, 1987; Hiday, 1988; Migneault et O'Neil, 1988; Brouillette et Paris, 1991; Gordon, 1993; Holstein, 1993) : des exigences plus grandes quant aux signes de 
danger pour soi-même ou pour autrui; des délais prolongés pour procéder; la mise en œuvre de procédures judiciaires ou quasi judiciaires; et le droit des personnes d'être représentées par un avocat (donc une procédure contradictoire). Ce second aspect des transformations en matière de santé mentale limitait les réponses susceptibles d'être apportées dans des cas de désorganisation, d'incohérence ou de signes de danger possible pour la personne elle-même ou pour autrui. Ce contexte, définissant les paramètres généraux de la dispensation des soins de santé tout autant que les conditions de réponses ponctuelles dans des cas perçus comme urgents, a contribué à rendre l'utilisation du système pénal particulièrement attrayante (Teplin, 1984; Freeman et Rosch, 1989; Laberge et Morin, 1995; Lamb et Weinberger, 1998).

Par ailleurs, d'autres transformations sociales importantes, dont les coupures sans précédent dans les programmes d'aide sociale et de logements sociaux, participaient à l'appauvrissement des groupes sociaux les plus démunis et à l'accroissement du phénomène des sans-abri. Or la condition d'itinérance, marquée entre autres par des expériences de victimisation, de dures conditions d'existence, des pratiques courantes de consommation excessive de drogue ou d'alcool, a contribué au taux non négligeable de troubles parfois graves de santé mentale.

Dans l'ensemble des bouleversements auxquels ces transformations ont donné lieu, des personnes souffrant de problèmes de santé mentale se sont retrouvées seules, sans soutien communautaire ou familial. Pour diverses raisons, les communautés qui devaient intégrer ces personnes n'ont pas réussi à faire face à ce défi (Dear et Wolch, 1987; Dorvil, 1988; Shabo, 1994; Comité de la santé mentale, 1997; Comité du bilan..., 1997). En conséquence, des personnes extrêmement fragiles et incapables de fonctionner socialement de façon adéquate se sont retrouvées sans soutien social, parfois même à la rue. Quant à celles qui vivaient dans l'itinérance, l'absence de domiciliation a représenté une des sources principales de leur difficulté chronique d'accéder aux services de santé mentale et de maintien des soins.

C'est dans ces conditions bien particulières qu'entrent en action le système judiciaire et particulièrement les policiers. En effet, ces derniers sont souvent appelés à intervenir auprès de personnes dont les comportements sont incohérents et semblent susceptibles de mettre leur sécurité ou celle des autres en danger. Devant la difficulté d'obtenir des soins, à cause de la résistance du personnel hospitalier ou des personnes ellesmêmes, les policiers peuvent intenter une poursuite judiciaire. Une telle 
action a généralement pour objectif de restaurer la paix et de protéger la personne ou son environnement (Bittner, 1967; Holley et ArboledaFlorez, 1988; Teplin et Pruett, 1992; Shabo, 1994; Green, 1997). Toutefois, la tendance à assimiler l'intervention de la police au caractère dangereux de la personne concernée rend l'accessibilité aux services encore plus difficile. Le policier qui fait appel à une ressource pour solliciter des services se bute souvent à des refus (James et Hamilton, 1992). Les intervenants qui répondent à l'appel des policiers occultent, dans bien des cas, le volet d'aide et d'assistance du travail policier, croyant ainsi que l'intervention policière s'adresse systématiquement à des individus dangereux, violents et dérangeants ${ }^{2}$.

Les services de police, qui interviennent en premier lieu, puis l'ensemble des agences du système de justice, particulièrement les prisons, se sont trouvés à prendre en charge des personnes très fragiles sur le plan psychologique lorsqu'elles ne vivent pas de pathologie importante (Lamb et Grant, 1982; Lefebvre, 1985; Adler, 1986; Hodgins et Côté, 1990; Lamb et Weinberger, 1998). Ces instances se trouvent démunies pour faire face à des personnes dont les besoins exigent des compétences particulières et qui, par ailleurs, ne présentent pas le profil habituellement associé à une délinquance « classique ».

\section{Les effets négatifs de la criminalisation}

Sur une base quotidienne, ce sont des critères socio-interactionnels qui servent à identifier « un problème » souvent qualifié de problème de santé mentale. La caractéristique centrale de cet ensemble de critères concerne la nature complètement inadéquate des comportements : l'incohérence, l'incapacité de s'orienter dans l'espace, les propos ou les comportements totalement déplacés, les conversations sans interlocuteurs, les comportements dangereux pour soi-même; un ensemble de façons de faire ou d'être qui transgressent des normes à propos desquelles il n'y a pas d'ambiguiité sur le plan social. Il s'agit de ce type de normes auxquelles on ne peut rattacher d'interprétations alternatives et dont la transgression entraine des doutes chez tous les observateurs quant à la capacité de fonctionner adéquatement de la personne. Nous désignons l'ensemble des comportements ou interactions comme des

2. Selon Way et al., 1993, les études tendent à montrer que les cliniciens sont influencés par la présence policière dans leur évaluation de la dangerosité des patients. Leurs propres résultats de recherche leur permettent d'avancer de nouveau cette hypothèse. 
«situations problèmes ». L'expression, en plus de sa référence normative, évoque la probabilité d'une intervention visant à faire disparaître le problème et à lui trouver une solution.

L'usage du système pénal pour répondre à ce type de situation est fondé sur une prémisse rarement explicitée par les intervenants ou par les chercheurs : la criminalisation générerait un bénéfice important, malgré son caractère parfois ambigu sur le plan légal. Les personnes seront prises en charge et soignées, généralement au cours d'une évaluation psychiatrique portant sur leur aptitude à subir leur procès. Cette prémisse n'est pas sans poser de problèmes : comment ces personnes sont-elles prises en charge ? Quelle est la nature des soins susceptibles (concrètement et légalement) d'être dispensés dans un contexte judiciaire ? Quelles sont les conséquences de cette stratégie pour les personnes ainsi prises en charge?

De nombreux policiers qui ont procédé à des arrestations de personnes très désorganisées ou apparemment malades étaient convaincus qu'en posant un tel geste, ces personnes seraient prises en charge et soignées (Laberge et al., 1995). Quels sont les scénarios possibles après que les policiers aient procédé à l'arrestation et souligné, dans leur rapport, la présence de problèmes de santé mentale ? Ces scénarios ont fait l'objet, en tout ou en partie, d'études (Hochstedler Steury, 1991; Laberge et al., 1996) permettant de mettre de l'avant les éléments suivants :

scénario 1 - les accusés ne sont pas perçus par les responsables du tri ${ }^{3}$ au tribunal comme devant être vus par le médecin, et ils sont tout simplement traités comme n'importe quel autre accusé;

scénario 2 - les accusés sont perçus comme étant suffisamment cohérents, même s'ils ne se portent pas bien, et ils ne seront pas évalués par un psychiatre pour leur aptitude à comparaître;

scénario 3 - les accusés sont perçus comme devant faire l'objet d'un examen de leur aptitude à comparaître, et ils sont détenus (généralement en prison) afin qu'un psychiatre puisse procéder à l'expertise.

Quel que soit le scénario mis en œuvre, l'intervention et la prise en charge par le système pénal ne sont pas sans conséquence. Si tous et chacun s'accordent à reconnaitre son caractère punitif, au moment de la prise en charge elle-même, peu de personnes se sont penchées sur les effets négatifs à court, moyen et long termes d'une telle mesure. D'abord

3. Selon les juridictions, il peut s'agir de policiers ou de procureurs. À Montréal, dans le cas de la cour municipale, ce sont des policiers, alors que dans le cas de la cour du Québec, chambre criminelle et pénale, il s'agit de procureurs de la couronne. 
à court terme, ces personnes seront certainement incarcérées. C'est d'ailleurs là le fondement même de la décision de judiciariser : placer la personne dans un contexte de surveillance où elle sera à même d'améliorer sa santé et d'obtenir des soins. Pour ces personnes, l'incarcération peut représenter des pertes beaucoup plus importantes que pour de nombreux autres citoyens. En effet, si elle dépasse quelques jours, l'incarcération peut signifier la perte d'un logement, la perte de ses rares biens, des difficultés accrues dans l'obtention de l'aide sociale, l'impossibilité de respecter certains rendez-vous. Cette question est toujours négligée dans les études sur les effets de l'emprisonnement, bien qu'elle soit mentionnée systématiquement par les dispensateurs de services. Les personnes qui manquent leur rendez-vous sont très mal perçues (mauvais patients, peu motivés, faisant perdre du temps à tout le monde) et elles risquent d'avoir des difficultés à obtenir de nouveaux rendezvous ou elles devront payer des frais de pénalité.

De façon générale, le fait d'avoir un casier judiciaire constitue un obstacle dans une diversité de situations : on peut penser, entre autres, à l'obtention d'un emploi ou d'un logement (Landreville et al., 1981; Marchetti, 1996). De façon plus particulière, une condamnation pénale devient aussi un obstacle pour accéder à certaines ressources de nature psychosociale (Laberge et Morin, 1995). En effet, une condamnation pénale constitue un stigmate qui se caractérise par la généralisation qu'il entraîne. C'est ainsi que les étiquettes de « délinquant », de « criminel », de « condamné » sont associées sans discrimination à la dangerosité potentielle, à l'agressivité, à la menace. Cet effet est à l'œuvre, quelle que soit la nature de la condamnation. Le terme étant général, on oublie que le système pénal prend en charge et condamne des comportements qui sont d'une extrême diversité et qui n'ont en commun que d'être condamnés par la loi (du vol à l'étalage à l'homicide volontaire). D'immenses différences sont ainsi occultées.

À moyen et à long termes, d'autres effets négatifs risquent de survenir lors de contacts pénaux subséquents (Porporino et Motiuk, 1993; Laberge et al., 1995). L'accusé peut être mis en détention provisoire parce qu'on craint que quelqu'un de socialement isolé ou qui a des problèmes d'insertion ne se présente pas à son procès. S'il est remis en liberté, il est possible qu'il ne se présente pas à son rendez-vous avec la justice pénale et qu'on émette un mandat d'arrestation contre lui. Dans ce dernier cas, s'il est trouvé coupable, ce qui est fort probable, on lui imposera souvent une ordonnance de probation incluant plusieurs 
conditions à respecter, dont celle de voir un thérapeute. Ici aussi, le nonrespect des conditions constitue une infraction qui provoquera une escalade dans l'engrenage pénal. Si son comportement bizarre, incohérent, est porté à l'attention de la police pendant qu'il est recherché pour avoir omis de se présenter à son procès, ou pour «bris de conditions », il sera probablement perçu comme un délinquant récalcitrant plutôt que comme quelqu'un qui a besoin d'aide. À plus long terme, une condamnation pénale contribue à définir la personne comme récidiviste et à entraîner l'escalade des mesures restrictives de liberté et de la sévérité des peines. Dans certains cas, les retours multiples dans le système pénal provoqueront une volonté de mise à l'écart de la part des intervenants pénaux. Une volonté qui se traduira par des peines d'incarcération extrêmement sévères par rapport à l'acte reproché.

En somme, dans les meilleures circonstances, si la stratégie qui consiste à utiliser le système pénal pour répondre à des besoins de prise en charge et d'intervention psychosociale réussissait (c'est-à-dire que les personnes obtiennent effectivement les soins dont elles ont besoin), elle est fort susceptible d'entraîner, à moyen terme, des effets négatifs dont le coût risque d'être beaucoup plus élevé que les bénéfices originaux.

\section{L'urgence psychosociale-justice}

\section{Le développement de l'UPSJ}

Au début des années 1980, à Montréal, tout comme dans d'autres régions du Québec, plusieurs acteurs du milieu de la santé et des services sociaux, de la justice pénale et des milieux communautaires, joignent leurs efforts autour de Tables de concertation santé mentale-justice. En 1986, un groupe de travail interministériel dépose un plan d'action destiné à répondre aux besoins de cette clientèle (Groupe de travail interministériel M.S.S.S - M.J.Q. - M.S.G.Q., 1986), qui aboutit, entre autres, à la mise sur pied de différents services dans les secteurs public et communautaire (service d'évaluation psychiatrique auprès des tribunaux judiciaires, Diogène $e^{4}$ Centre de psychiatrie légale de Montréal) (Table de concertation psychiatrie-justice de Montréal, 1989), et à la signature d'un protocole interministériel (Santé et Services sociaux et Sécurité publique). Ce protocole comprenait la signature d'ententes avec des ressources d'héber-

4. Centre communautaire en psychiatrie-justice. 
gement communautaires afin d'assurer, de façon un peu plus stable, l'intégration de ces personnes dans des ressources largement sous-financées. Cet événement a constitué un moment important dans la reconnaissance, sociale et politique, d'une difficulté persistante à répondre aux besoins de cette population, particulièrement en termes d'accessibilité à des services, et de la volonté des organismes rattachés à ces ministères et des ressources communautaires de faire face à la situation.

Durant cette même période, cette problématique faisait l'objet de travaux de recherche (Lefebvre, 1985; Laberge et al., 1991; Laberge et Morin, 1992; Régie régionale de la santé et des services sociaux de Montréal-Centre, 1994a, b, 1996). Le projet d'urgence psychosociale était alors mis de l'avant et a cheminé jusqu'à l'ouverture, en octobre 1996, de l'UPSJ. La Régie régionale de Montréal-Centre a confié le mandat de coordonner l'implantation de ce service au CLSC des Faubourgs. L'UPSJ est une unité multipartite sous la supervision d'un consortium qui regroupe des « acteurs-terrain » représentant les différents organismes partenaires : le réseau des ressources communautaires, le Centre de crise (Transit - Ombrelle), Diogène, le Centre Dollard-Cormier, le SPCUM, les représentants du ministère de la Justice, du ministère de la Sécurité publique et des hôpitaux, le CLSC des Faubourgs, le Centre de psychiatrie légale de Montréal, l'Institut Philippe Pinel, l'UQAM, Urgences-santé, la Ville de Montréal et la Régie régionale de Montréal-Centre. Ce consortium est responsable du maillage des interventions entre l'équipe d'intervenants de l'UPSJ et toutes les autres ressources ou services appelés à intervenir avec la ressource et auprès de la clientèle visée. L'équipe d'intervenants (au nombre d'une douzaine) provient de ressources communautaires et d'organismes publics du réseau de la santé et des services sociaux et du réseau de la justice pénale.

L'UPSJ vise la clientèle «psychiatrie-justice » qui a été définie comme

[une personne] de 18 ans et plus ayant un problème sévère de santé mentale pouvant inclure des troubles de personnalité auxquels peuvent s'associer des comportements délictueux [...]. Lors de l'intervention, cette personne peut être agitée, désorientée dans l'espace et le temps ou délirante [...], se faire menaçante, dérangeante, ou être sous le coup d'une intoxication quelconque [...], peut aussi avoir cessé depuis quelque temps sa médication, d'où sa désorganisation ponctuelle (CLSC des Faubourgs, 1996).

Au plan de l'intervention, le mandat de l'UPSJ comporte deux volets distincts mais intimement liés : un volet d'intervention sociojudiciaire qui vise une 
diminution significative ou espacement des situations où il y a judiciarisation du comportement délictueux ou, tout au moins, la non-incarcération; un volet d'intervention psychosociale dont le but est un apprivoisement de la personne aux prises avec un problème de santé mentale-justice dont la tendance est d'être réfractaire à l'aide et au soutien qu'on lui offre [...], un arrimage rapide et ponctuel du besoin de la personne avec les services et les ressources appropriés existants dans son environnement [...] et une intervention, le plus tôt possible en début de crise, dans le but de prévenir ou d'éviter l'exclusion de la personne de son environnement (CLSC des Faubourgs, 1996).

Quant au fonctionnement de l'équipe d'intervenants, il est conçu pour permettre la réalisation de multiples activités que nous pourrions classer selon trois modalités distinctes d'intervention. La principale consiste à répondre à des appels d'urgence qui impliqueront régulièrement le déplacement d'intervenants sur les lieux d'événements. Dans ce cas particulier, il s'agit pour l'essentiel de désamorcer une crise, d'apporter un support aux personnes impliquées, qu'il s'agisse d'autres intervenants ou des personnes en conflit, de proposer une solution à court terme (accompagnement dans une ressource d'hébergement, à l'hôpital, etc.), et enfin d'assurer le suivi de l'affaire jusqu'à son dénouement ou son relais vers une autre ressource de prise en charge (ressource d'hébergement, hôpital, centre de désintoxication, etc.). Pour réaliser ces activités, l'équipe d'intervenants est répartie sur trois quarts de travail afin d'offrir le service 24/7. En général, les intervenants travaillent en groupe de deux. La deuxième modalité d'intervention correspond au service à la cour. En effet, l'UPSJ offre un service sur place, à la cour municipale de Montréal et à la cour du Québec à Montréal. Un intervenant est assigné à ce travail et sa tâche consiste principalement à répondre à des demandes d'évaluation de justiciables présentant des signes des troubles mentaux, et qui sont susceptibles de faire l'objet de prise en charge ou de suivi par d'autres types de services; le travail de l'intervenant vise à éviter une prolongation inutile de la détention provisoire ou encore une peine d'incarcération. Enfin, une troisième modalité d'intervention correspond à la promotion du programme de l'UPSJ et à la sensibilisation des partenaires à la problématique de la criminalisation de la maladie mentale. Ces dernières activités prennent la forme de visites des différentes ressources ou services partenaires d'UPSJ effectuées par les intervenants de l'équipe. En général, chaque ressource est visitée au moins une fois par année depuis l'ouverture du service.

En somme, le concept d'UPSJ repose sur la reconnaissance d'un problème sérieux de jonction entre les services dans la production du 
phénomène de criminalisation de la maladie mentale. Le programme est ainsi défini comme

un outil de coordination favorisant la prévention et la résolution des problèmes de santé mentale-justice dans la communauté de même que la prise en charge des personnes par les services réguliers de santé et de services sociaux et les organismes communautaires [...], un service de support à l'intervenant policier appelé à intervenir sur les lieux d'un événement et à l'intervenant social confronté à une situation de crise dans son milieu de travail [...], un support au procureur et à l'intervenant des Services correctionnels qui doivent s'assurer que la personne aux prises avec un problème de santé mentale et ayant agi de façon délictueuse reçoive les services de santé appropriés (CLSC des Faubourgs, 1996).

La recherche à l'UPSJ :

quelques indications méthodologiques

L'UPSJ est appelée à intervenir dans une variété de situations qui comportent toutes une dimension d'urgence. Par ailleurs, elle assure un mandat explicite de jonction entre les services et les individus qui délimite en quelque sorte l'ampleur ou l'étendue de l'intervention. Cette double réalité du programme UPSJ détermine, dans une large mesure, le contenu de l'information susceptible d'être accessible, validée, et conservée sur chacune des interventions. En effet, dans le contexte où $55 \%$ des dossiers UPSJ sont traités dans une période de moins de 24 heures, la cueillette d'information par les intervenants sur le terrain est objectivement limitée. Par conséquent, l'analyse des interventions UPSJ à partir des dossiers cliniques dépend de ce contexte particulier de leur pratique.

Dans la recherche que nous poursuivons à l'UPSJ, nous avons retenu une approche méthodologique mixte, à la fois qualitative et quantitative, ainsi que différentes sources de données et plusieurs stratégies de cueillette. Dans cet article, nous présentons quelques résultats préliminaires de l'étude des dossiers de l'UPSJ ${ }^{5}$. Pour ce volet, nous avons

5. Considérant le contexte d'intervention de l'UPSJ (fréquentes situations d'urgence et de crise), l'absence de domiciliation parmi une part importante de la clientèle UPSJ et l'approche rétrospective dans l'analyse des dossiers, il nous était impossible d'obtenir le consentement des clients de l'UPSJ avant de consulter leur dossier. Toutefois, notre devis de recherche a été approuvé par le comité d'éthique de l'UQAM. De plus, nous avons obtenu l'autorisation de consulter des données nominatives pour fins de recherche de la Commission d'accès à l'information qui nous impose plusieurs conditions de protection de la confidentialité et l'obligation de détruire les données dans le délai prescrit. 
retenu une approche quantitative qui se fonde sur une cueillette d'informations systématique dans les dossiers d'intervention de l'UPSJ ${ }^{6}$. Nous avons procédé à la cueillette d'informations sur toutes les demandes adressées à l'UPSJ, que ces demandes aient été considérées ou non comme relevant du service, entre le 15 octobre 1996 et le 30 avril 1998. Au cours de cette période, 1329 demandes ont été adressées à l'UPSJ concernant 970 personnes. Pour la saisie des données, nous avons utilisé un logiciel de bases de données relationnelles nous permettant de traiter les interventions de l'UPSJ selon deux logiques distinctes : une première dont l'unité d'analyse correspond à la personne qui est l'objet d'une intervention; une deuxième dont l'unité d'analyse correspond à une demande adressée à l'UPSJ.

\section{Des résultats préliminaires}

Nous examinerons les activités de l'UPSJ sous quatre angles distincts :

1) Quel est le profil des personnes qui font l'objet d'une demande d'intervention UPSJ et qui sont susceptibles de se qualifier ou non pour une intervention UPSJ, et de faire ou non l'objet d'une judiciarisation?

2) Quelle est la nature des situations problèmes à l'origine des demandes d'intervention?

3) Quel est le profil des sources de références à l'UPSJ et quelle est la nature de leur demande?

4) Quelles sont les réponses apportées aux demandes adressées à l'UPSJ ?

Le profil de la clientèle UPSJ

La clientèle "psychiatrie-justice » à qui s'adresse le projet UPSJ a toujours été identifiée comme une population problématique, mais pour différentes raisons et selon différents points de vue. Souvent caractérisée par des problématiques multiples (maladie mentale, toxicomanie, itinérance, isolement social), par des problèmes d'accessibilité aux services, ou parfois par le fait de sa circulation entre différentes agences et services de différents systèmes (pénal, psychiatrique, santé, services

6. En concertation avec l'équipe d'intervenants, nous avons élaboré une grille de cueillette d'information dans laquelle certaines rubriques contiennent des catégories prédéfinies alors que d'autres correspondent à des champs ouverts. Cet outil est utilisé par les intervenants et est complété par des notes cliniques. 
sociaux, réseau communautaire), cette population est demeurée difficile à saisir. Cet aspect de l'analyse vise à tracer le profil de la clientèle pour laquelle une demande d'intervention a été sollicitée à 1'UPSJ. Cette donnée est essentielle pour saisir les problématiques susceptibles d'être rattachées à la population desservie par la ressource.

Le portrait qui se dégage tend à montrer que la clientèle de 1'UPSJ présente les caractéristiques communes aux populations «psychiatriejustice ». Elle est composée majoritairement d'hommes (70\%), mais la proportion de femmes est importante. À cet égard, elle est supérieure au ratio habituel des populations pénales et correspond ainsi davantage au profil « psychiatrie-justice » (Laberge et al., 1991; Brinded et al., 1996; Green, 1997) ${ }^{7}$. L'âge constitue un second indicateur de ce profil. Au moment de la première intervention, l'âge moyen est de 40 ans. Découpé en tranches d'âge, on constate que $58 \%$ des hommes et $71 \%$ des femmes avaient plus de 35 ans. Dans le seul groupe des 50 ans et plus, ce pourcentage atteint pour les hommes $18 \%$ et $29 \%$ pour les femmes ${ }^{8}$. En ce qui a trait à l'isolement social, la très grande majorité de la clientèle (87\%) est seule et une forte proportion est célibataire (73\%). Un profil d'extrême pauvreté et d'itinérance émerge clairement. D'une part, la majorité est bénéficiaire de l'aide sociale (69\%) ou encore sans aucun revenu (13\%). D'autre part, au moment de l'intervention, $25 \%$ des personnes sont sans adresse et $16 \%$ vivent dans des refuges pour personnes itinérantes. Lorsqu'on examine l'importance de cette dimension selon le sexe des personnes, la proportion des femmes vivant dans des refuges ou étant sans adresse au moment de l'intervention UPSJ est presque la même (39\%) que celle des hommes dans la même situation (42\%).

$\mathrm{Au}$ plan de la santé mentale, la trace d'antécédents psychiatriques ${ }^{9}$ parmi la clientèle UPSJ est très importante. On note de tels antécédents

7. Dans l'étude que nous avons menée à la cour municipale de Montréal en 1990 sur le traitement judiciaire des personnes connaissant des problèmes de santé mentale, la proportion hommes/femmes était exactement la même.

8. Comparativement aux données de la cour municipale de Montréal en 1990 (Laberge et al., 1991), la proportion d'individus âgés de 35 ans et plus est, dans la population de l'UPSJ, plus importante de $11 \%$.

9. Les antécédents psychiatriques sont entendus dans un sens très large et se réferent à tout contact avec des services de santé mentale (hospitalisation, suivi en clinique externe, etc.). Cette donnée ne peut être validée par les intervenants de l'UPSJ. Il n'existe pas de banque unique permettant de retracer la nature et l'étendue d'une telle expérience. Dans les dossiers cliniques de l'UPSJ, cette information peut provenir de sources diverses : le client, son entourage, les intervenants des ressources, le personnel hospitalier où a déjà séjourné le client, etc. 
chez $80 \%$ de la population. Quant aux données judiciaires ${ }^{10}$, elles révèlent que $55 \%$ des interventions UPSJ concernent des individus qui comptent des antécédents de condamnation (DACOR et/ou FPS) ${ }^{11}$, dont un nombre important parmi ceux-ci $(43 \%)$ a déjà été condamné à une peine d'incarcération. Le portrait des antécédents judiciaires selon le sexe des personnes attire l'attention sur le fait que, même si les femmes ont un profil pénal moins lourd que celui des hommes, elles sont beaucoup plus nombreuses à présenter cette caractéristique que dans l'ensemble des populations pénales en général. Ainsi, $42 \%$ des femmes ont des antécédents de condamnation comparativement à $61 \%$ des hommes. Au moment de la demande d'intervention à l'UPSJ, près d'une demande sur cinq $(18 \%)$ concerne des individus qui ont un dossier actif dans le système pénal (cause pendante, probation, libération conditionnelle, etc.).

Les situations problèmes ou incidents

L'intervention d'un service tel l'UPSJ suppose la présence d'une "situation problème », d'un incident, ou l'appréhension d'un tel problème dans un laps de temps relativement court, ou encore la prévention de situations susceptibles de mener à des conflits interpersonnels et à la judiciarisation. Cette diversité de situations n'implique pas toujours la même expertise, la même intervention, ni les mêmes partenaires. Elles ont des implications spécifiques du point de vue des démarches de déjudiciarisation selon que le processus est ou non enclenché, selon l'étape à laquelle l'affaire est rendue devant le tribunal. Il est donc nécessaire de distinguer au départ à quelle catégorie appartiennent les situations qui font l'objet d'une demande d'intervention à l'UPSJ.

Une catégorisation des situations problèmes a été réalisée par l'équipe de recherche suite à une analyse qualitative des descriptions de ces incidents inscrites au dossier clinique par les intervenants de l'UPSJ (tableau 1). Plus du quart (28\%) des incidents sont rapportés comme étant des

10. Les données judiciaires de la clientèle UPSJ ont été recueillies dans deux sources officielles (DACOR et FPS) et compilées par l'équipe de recherche. Cette compilation tient compte de la situation de chaque individu au moment de la demande d'intervention UPSJ.

11. Les condamnations inscrites dans le FPS sont les condamnations à des poursuites judiciaires par voie de mise en accusation pour des actes criminels, mais également toutes celles à propos desquelles l'accusé a fourni ses empreintes digitales. Les condamnations inscrites dans l'historique judiciaire de DACOR sont toutes les condamnations qui ont donné lieu à une prise en charge par les Services correctionnels du Québec; ce qui veut dire toutes les condamnations à des peines d'incarcération gérées par les Services correctionnels du Québec ainsi que toutes les condamnations comprenant une ordonnance de probation. 
conflits entre des proches ${ }^{12}$. Ces comportements peuvent aussi bien avoir pris comme cible la personne elle-même ou ses biens. De la même manière, les comportements agressifs contre des étrangers, qui représentent $23 \%$ de l'ensemble des situations problèmes, comprennent autant les menaces de toute nature, les coups, que les bris de biens appartenant aux étrangers. Le poids relatif de ces deux catégories, qui regroupent la moitié des incidents, souligne ici l'importance des conflits interpersonnels dans la problématique de la criminalisation de la maladie mentale. Quant à la troisième catégorie en importance, elle regroupe les comportements d'incohérence qui ont en commun leur caractère bizarre, totalement inapproprié au contexte : elle compte pour $18 \%$ des demandes d'intervention. Ces situations donnent lieu à une attention particulière au point où une intervention formelle s'engage. L'importance de l'itinérance dans la configuration de cette population n'y est pas étrangère. On remarque par exemple que, dans ce type de situations problèmes, $50 \%$ des demandes concernent des sans-abri ou des personnes fréquentant les refuges. Enfin, la dernière catégorie en importance regroupe les situations décrites comme de la prévention, dans la mesure où il n'y a pas d'incident précis mais où on craint un passage à l'acte. Cette catégorie compte $14 \%$ des demandes d'intervention.

Les sources de référence et les demandes d'intervention

Les 1329 demandes adressées à l'UPSJ concernent 970 personnes. En moyenne, l'UPSJ a reçu 73 demandes par mois. Toutefois, les demandes ont augmenté au cours de la période de l'étude (15 octobre 1996 au 30 avril 1998), ce qui correspond à la consolidation du service ainsi qu'à l'élargissement du territoire initialement défini.

L'étape à laquelle surviennent les demandes représente un potentiel d'action en termes de non-judiciarisation ou déjudiciarisation pour l'UPSJ puisqu'elle se situe en prévention, avant une intervention policière, dans 42 \% des demandes; à l'étape de l'intervention policière dans

12. Dans cette étude, la catégorie des proches comprend non seulement les membres de la famille, les amis, l'entourage, mais également un propriétaire d'immeuble où habite la personne par exemple, un voisin, un résident ou le personnel d'une ressource fréquentée par le client UPSJ. Il faut souligner que dans le tableau 1, nous avons regroupé la catégorie « conflit entre les proches » $(\mathrm{n}=281)$ et « conflits conjugaux » $(\mathrm{n}=90)$, catégories qui sont présentées séparément dans le tableau 2. Quant à la catégorie des étrangers, elle comprend les inconnus des personnes en cause, les policiers appelés sur les lieux, même si ceux-ci peuvent connaître la personne. 
TA B LEA U 1

Distribution des types de situations problèmes

\begin{tabular}{|l|c|c|}
\hline \multicolumn{1}{|c|}{ Situations problèmes } & Fréquence & $\%$ \\
\hline Les conflits entre des proches & 371 & 28 \\
\hline $\begin{array}{l}\text { Les comportements agressifs contre des } \\
\text { étrangers }\end{array}$ & 306 & 23 \\
\hline Les comportements incohérents & 236 & 18 \\
\hline La prévention de comportements appréhendés & 178 & 14 \\
\hline Les autres situations ou comportements & 72 & 5 \\
\hline Les événements d'expulsion & 62 & 5 \\
\hline Les comportements suicidaires & 62 & 5 \\
\hline L'absence de situation problème & 35 & 3 \\
\hline Total & $\mathbf{1 3 2 2}$ & $\mathbf{1 0 0} \%$ \\
\hline
\end{tabular}

Données manquantes : 7

$33 \%$ des cas, ou encore à la cour avant la première comparution dans $15 \%$ des cas.

Deux sources principales approvisionnent l'UPSJ avec respectivement $34 \%$ et $33 \%$ des renvois. Ce sont les ressources du milieu ${ }^{13}$ et la police. Les autres demandes adressées au service proviennent de la cour du Québec à Montréal, chambre criminelle et pénale (15\%), de la cour municipale de Montréal (8\%), et enfin d'une variété d'autres sources $(11 \%)^{14}$.

Deux types principaux de demandes sont adressés à UPSJ : l'intervention $(69 \%)$ et les demandes de conseils et d'information (28\%).

L'analyse des situations problèmes combinée aux éléments du contexte dans lequel survient la demande nous permet de constater une différenciation des situations selon la source de référence à l'UPSJ. Ces situations ne se distribuent pas aléatoirement entre les différentes catégories de sources de référence à UPSJ. Il existe une tendance à la spécialisation dans le renvoi. Ainsi, les différentes catégories de situations problèmes sont associées à une ou deux sources de référence (tableau 2).

Un second élément participe à la construction de cette spécialisation dans le renvoi. Il s'agit de la domiciliation. En fait, nous croyons que cette condition confere un statut particulier aux individus et contribue à définir

13. Ces ressources comprennent principalement des ressources pour personnes seules et itinérantes, le CLSC des Faubourgs et une diversité de CLSC et de centres hospitaliers.

14. Cette catégorie comprend une très grande diversité de sources de références: concierge, réceptionniste d'hôtel, propriétaire de restaurant ou d'immeuble à logement, etc. Ce sont, pour la majorité des cas, des appels effectués à titre de citoyen. 
l'identité des personnes et des situations problèmes. Certains individus, en raison de leur condition de domiciliation, seront perçus comme des cas relevant de l'UPSJ, alors que, pour d'autres sources de références, cette condition ne permettra pas de se qualifier pour une intervention UPSJ. Ainsi, nous pouvons dégager une association relativement importante entre cette condition de domiciliation et la source de référence à l'UPSJ. On constate que parmi les gens qui déclarent habiter dans un logement, une proportion importante provient de la cour du Québec (27\%), alors qu'elle ne contribue que pour $16 \%$ de toutes les demandes. Parmi les cas sans adresse ou en refuge, on remarque nettement l'importance des ressources comme source de renvoi. Plus de la moitié (54\%) des appels concernant cette catégorie proviennent des ressources. Enfin, lorsqu'on est chambreur, les probabilités d'être référé par la police sont plus importantes que celles de l'être par d'autres sources.

Les réponses

Comme nous l'avons déjà mentionné, l'UPSJ a un mandat large qui ne se limite pas à l'intervention individuelle auprès d'un client en besoin d'aide. Il comporte une dimension d'interface et de support aux organismes et aux ressources qui suppose, en dehors des appels courants de services, des activités d'information et de concertation. Par ailleurs, cette intervention poursuit un objectif clair de déjudiciarisation qui comporte également un double volet : éviter la judiciarisation et l'incarcération; éviter la détérioration de la situation de la personne pour contrer le phénomène des retours multiples (devant les tribunaux et dans les urgences psychiatriques). La mise en œuvre de ce mandat donne à l'activité de l'UPSJ une configuration complexe quant aux différentes modalités d'intervention qui peuvent être échafaudées : des activités de prévention (en co-intervention ou non); des interventions en situation de pré-crise ou de crise; des activités de relais entre les ressources; des suivis de cas; de l'accompagnement; des activités de support (client, proches ou ressources); de la référence et de l'orientation. Mais ce qui fait la spécificité de l'UPSJ, c'est que son mandat de déjudiciarisation délimite, dans la réalité, le cadre de ses interventions. Celles-ci s'élaborent au cours de l'un ou l'autre des nœuds décisionnels suivants :

- une ressource intervient et décide de ne pas faire appel à la police si l'UPSJ peut faciliter rapidement une prise en charge d'une autre nature; 


\begin{tabular}{|c|c|c|c|c|c|c|c|}
\hline $\begin{array}{l}\overrightarrow{0} \\
\stackrel{\Phi}{ \pm}\end{array}$ & $\begin{array}{l}\text { 辛 } \\
\text { 离 } \\
\text { 品 }\end{array}$ & 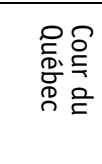 & 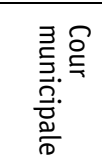 & 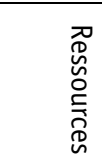 & $\frac{\overline{0}}{\stackrel{\bar{D}}{0}}$ & & \\
\hline 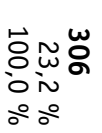 & 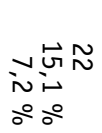 & 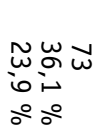 & 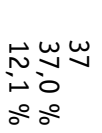 & $\begin{array}{l}\vec{\omega} 0 \\
\dot{\omega} \omega \\
\circ \\
\circ \\
\circ\end{array}$ & $\begin{array}{l}\hat{w} \omega_{0}^{\omega} \underset{\omega}{\omega} \\
v_{\infty}^{\infty} \\
\circ \circ 0\end{array}$ & 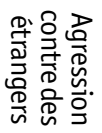 & \multirow{10}{*}{ 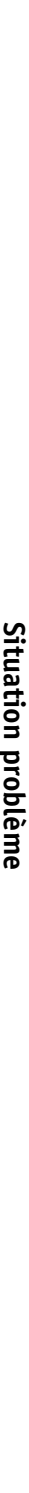 } \\
\hline 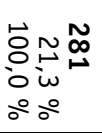 & $\begin{array}{l}\vee v i n \\
\infty:{ }^{n} \\
\circ \circ\end{array}$ & $\begin{array}{l}\tilde{\omega} \underset{w}{w} g \\
\infty \tilde{\nu} \\
\circ \circ\end{array}$ & 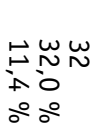 & 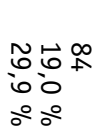 & 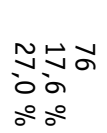 & 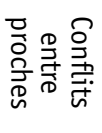 & \\
\hline 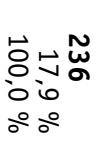 & 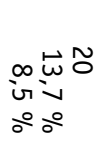 & $\begin{array}{l}\circ 01 \\
\text { 우 } \\
\circ \circ\end{array}$ & $\begin{array}{l}w \cup v \\
0: 0 \\
\circ \circ \\
\circ:\end{array}$ & 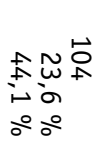 & 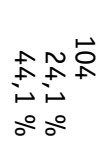 & 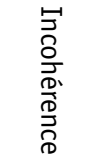 & \\
\hline 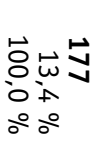 & 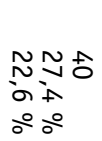 & $\begin{array}{l}\omega \omega \sigma \\
i 0 \\
\circ \circ \\
\circ\end{array}$ & $\begin{array}{l}N \in G \\
\infty \quad 0 \\
\infty \circ \\
\circ \circ\end{array}$ & 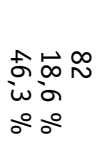 & 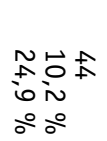 & 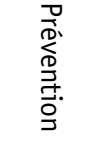 & \\
\hline 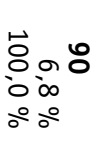 & $\begin{array}{l}\omega \sim \omega \\
\omega \sim \sim \\
\circ \circ \\
\circ \circ\end{array}$ & 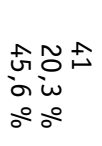 & 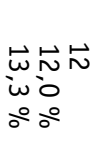 & $\begin{array}{l}\text { on } 0 \\
\text { :0 } \\
\circ \therefore \\
\circ \circ\end{array}$ & $\begin{array}{l}N \underset{N}{N} \tilde{N} \\
\infty \infty \\
00 \\
0\end{array}$ & 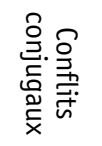 & \\
\hline 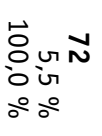 & 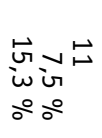 & 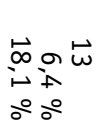 & $\begin{array}{l}\infty \circ a \\
\text { wo } \\
\circ \circ \\
0\end{array}$ & $\begin{array}{l}w a \tilde{v} \\
\text { vin } \\
\circ \circ \circ\end{array}$ & $\begin{array}{l}\tilde{O} w \vec{v} \\
\infty i v \\
\circ \therefore \\
\circ\end{array}$ & 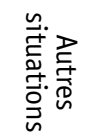 & \\
\hline 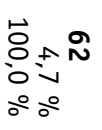 & $\begin{array}{l}\tilde{F}_{\infty} \omega \\
06 \\
0 \\
\circ \\
0\end{array}$ & 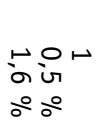 & $\begin{array}{l}\vdash \bullet \bullet \\
6 \circ 0 \\
\circ \circ\end{array}$ & 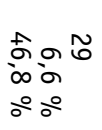 & 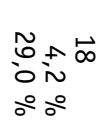 & $\begin{array}{l}\frac{\pi}{x} \\
\frac{x}{0} \\
\frac{5}{y} \\
\frac{0}{y}\end{array}$ & \\
\hline 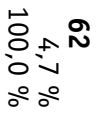 & 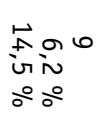 & & & 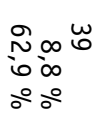 & 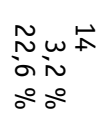 & $\begin{array}{l}\text { 气. } \\
\text { ลั. }\end{array}$ & \\
\hline 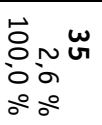 & 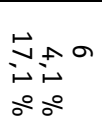 & & & 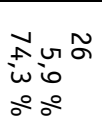 & $\begin{array}{l}\infty 0 w \\
o: v \\
\circ \circ \\
\circ\end{array}$ & 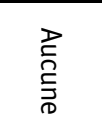 & \\
\hline 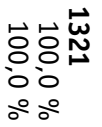 & 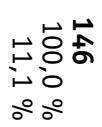 & 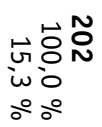 & 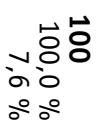 & 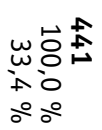 & 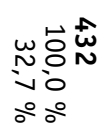 & $\begin{array}{l}\overrightarrow{1} \\
\stackrel{\Phi}{ \pm}\end{array}$ & \\
\hline
\end{tabular}


- la police intervient mais peut décider de ne pas porter d'accusation si une prise en charge d'une autre nature est rapidement disponible;

- l'affaire est judiciarisée, mais la disponibilité de mesures sociales ou thérapeutiques pourrait faire en sorte d'éviter la détention provisoire;

- la personne est condamnée mais le juge peut rejeter la peine d'incarcération si une solution de rechange est proposée $\mathrm{e}^{15}$.

Dans de nombreuses situations, l'implication réelle ou potentielle de l'appareil judiciaire impose ainsi certaines contraintes à l'intervention de l'UPSJ qui ne seront pas sans conséquences sur la façon dont on concevra l'urgence, la prévention et le suivi. Par ailleurs, cette dimension pourrait également constituer un contexte favorisant l'usage d'une mesure implicite ou explicite de contrôle à l'intérieur de laquelle s'exerce le pouvoir de contraindre un individu à se conformer aux attentes de groupes d'intervenants (Lovell, 1996).

Une première donnée importante montre que, dans $30 \%$ des demandes, l'UPSJ est en contact exclusivement avec des services et ne rencontre pas directement le client, ce qui reflète la part considérable de l'activité de soutien de l'UPSJ envers d'autres services.

De tous les appels adressés à l'UPSJ, les deux tiers se règlent en une journée ou moins. L'allongement de la durée d'intervention de l'UPSJ s'explique entre autres par le recours à l'hospitalisation au cours de l'intervention de l'UPSJ pour lequel nous avons constaté une forte association; ces hospitalisations surviennent dans $19 \%$ des appels. Alors que les dossiers ouverts pendant cinq jours et plus ne représentent que $30 \%$ des appels, dans le cas des hospitalisations, le groupe des demandes où l'intervention se prolonge à cinq jours et plus atteint $59 \%$ des cas. Par ailleurs, le prolongement à plus de cinq jours pourrait également être relié au phénomène des retours multiples à l'UPSJ. Si c'était le cas, comme les données le laissent entrevoir, cette situation pourrait traduire la persistance de problèmes de renvoi et de prise en charge d'un sousgroupe parmi la clientèle psychiatrie-justice ${ }^{16}$.

15. Dans le cas des deux derniers moments décisionnels, l'activité de l'UPSJ ne permet pas d'en rendre compte. La saisie de cette information supposerait un dispositif de recherche qui se situe en aval de l'intervention UPSJ, ce qui n'a pas été possible jusqu'à présent.

16. Nous consacrerons un prochain article à l'important phénomène des retours multiples à l'UPSJ. 
Quant à la judiciarisation ${ }^{17}$, elle survient dans $11 \%$ des cas, ce qui n'inclut pas les demandes qui surviennent à l'étape du tribunal, c'est-àdire les demandes pour lesquelles la judiciarisation était déjà en cours au moment de l'appel (24\% des cas). Or, la judiciarisation est fortement associée au type de situations problèmes. Ainsi, lorsque l'incident réfere à une situation d'agression contre un étranger, la judiciarisation survient dans $27 \%$ des cas, comparativement à $17 \%$ dans le cas des conflits entre proches; à $12 \%$ dans les cas de conflit conjugal; à $4 \%$ dans les cas d'incohérence; à $3 \%$ dans les cas d'expulsion; à $3 \%$ dans les cas de prévention; à $2 \%$ dans les cas de comportements suicidaires; et enfin, à $19 \%$ dans les cas des autres situations problèmes.

\section{Discussion}

Les résultats préliminaires nous permettent d'affirmer que l'UPSJ rejoint les populations ciblées par le projet initial. On remarque en effet que la clientèle est très isolée, qu'une part importante apparait instable sur le plan résidentiel, que les individus ont déjà eu des contacts avec des services de santé mentale dans le passé et qu'une proportion non négligeable a déjà été trouvée coupable d'infractions. De plus, nous avons vu qu'un nombre important d'appels concerne des individus en contact avec le système pénal au moment de la demande de service à l'UPSJ. Connaissant l'effet d'un tel marqueur dans les décisions des agences du système pénal, cette donnée peut réduire la marge de manœuvre de l'UPSJ dans ses objectifs de déjudiciarisation; elle peut aussi représenter une condition de négociation poussant l'individu à se conformer aux attentes des intervenants impliqués. Il s'agit en fait d'un des éléments du contexte d'intervention qui peut contribuer à l'usage d'une des formes de contrôle social : la participation contrainte, où la menace de sanction est utilisée pour inciter la participation au traitement (Lovell, 1996).

Concernant les sources de référence à l'UPSJ, nous sommes étonnés de constater la faible demande provenant de la cour municipale de Montréal. Ce tribunal traite depuis longtemps un volume considérable d'affaires mineures dont une part importante a été traditionnellement

17. La question de la judiciarisation est difficile à saisir à partir des seuls dossiers de l'UPSJ, puisque cette décision n'appartient pas à l'UPSJ et qu'elle peut être prise à différentes étapes d'un processus d'intervention qui peut impliquer ou non la présence de l'UPSJ. D'autres dispositifs de recherche devront être mis en œuvre pour traiter plus adéquatement cette question. 
associée à des individus soupçonnés de souffrir de troubles mentaux. Au début des années 1990, le médecin de ce tribunal effectuait en moyenne 22 examens sommaires ${ }^{18}$ par mois, à la demande des policiers du quartier général dans 96 \% des cas (Laberge et al., 1991 : 59). Aujourd'hui, la cour municipale ne fait appel à l'UPSJ en moyenne que 5,6 fois par mois. La réorganisation des services à la cour et le virage communautaire du service de police de la Communauté urbaine de Montréal sont des facteurs qui devraient être considérés, avec l'implantation de l'UPSJ, dans l'analyse du phénomène de la judiciarisation de la maladie mentale à Montréal.

S'il y a aujourd'hui un large consensus quant à l'utilité sociale de l'UPSJ, ce nouveau programme doit maintenant s'assurer d'une forte consolidation de son action avec ses partenaires-terrain. Les incidents impliquant des individus ayant des problèmes de santé mentale ne sont pas susceptibles de diminuer. Au contraire, certaines circonstances risquent d'accroître la demande à l'UPSJ. Nous pensons en particulier à la nouvelle vague de désinstitutionnalisation qui s'engage, à la réforme de la loi sur l'internement civil involontaire qui réserve aux policiers un pouvoir accru dans le processus d'internement et, par conséquent, à la multiplication de leurs contacts avec les personnes souffrant de problèmes de santé mentale.

\section{Conclusion}

Les pratiques qui donnent corps à la déjudiciarisation sont complexes et il est impossible de les comprendre à travers le seul biais des interventions de l'UPSJ. Les données préliminaires présentées dans ce texte permettent toutefois de faire quelques constats à ce chapitre. D'abord, la judiciarisation de situations problèmes associées à la maladie mentale constitue toujours une option non négligeable. Nous en observons entre autres la trace dans le nombre important de demandes issues des tribunaux. Ensuite, l'importance de la demande adressée à l'UPSJ, particulièrement en situation de pré-crise et de crise, signale sans nul doute le besoin d'une ressource spécialisée en santé mentale-justice et souligne une volonté, exprimée par la diversité des sources de références à l'UPSJ,

18. L'examen sommaire vise à émettre un avis sur l'aptitude à comparaitre des justiciables présentant des problèmes de santé mentale. 
de répondre à ce type de situations problèmes par d'autres voies que la voie pénale.

Une question fondamentale reste cependant en suspens. Existe-t-il, dans la communauté, des ressources suffisantes et adaptées pour prendre véritablement le relais de l'UPSJ ? Actuellement, l'UPSJ apparaît jouer un rôle clé en amont, c'est-à-dire en créant des conditions permettant d'éviter l'entrée dans le système pénal (désamorcer des crises, orienter vers des ressources plus appropriées, faire de la médiation, etc.). Cependant, le succès du service repose également sur la capacité du milieu de répondre à son tour à la demande de l'UPSJ, c'est-à-dire à prendre en charge et à offrir des soins adéquats et continus, à favoriser la réintégration sociale, à fournir un support véritable pour éviter les désorganisations répétées, la dégradation des situations et le retour dans le circuit de l'intervention d'urgence avec son risque de judiciarisation. Or, les ressources susceptibles d'effectuer ce relais ont fait l'objet de fortes compressions budgétaires (ressources institutionnelles) ou encore disposent de moyens réduits pour opérer faute de financement adéquat (ressources communautaires). Comme l'ont bien démontré plusieurs études (Peyrot, 1982; Brown, 1989; Lovell, 1996), un contexte de rareté des ressources génère des pratiques informelles de tri et de sélection des clientèles, pratiques auxquelles sont extrêmement vulnérables les clientèles psychiatrie-justice. Ainsi, s'il devait y avoir absence de réponse de la part du milieu, l'UPSJ pourrait rapidement être perçue comme ne pouvant pas véritablement remplir son mandat. En d'autres termes, la confrontation répétée avec les mêmes individus, sur des périodes relativement courtes, par les organismes qui réferent actuellement des personnes à l'UPSJ (on pense en particulier à la police), pourrait être interprétée comme une incapacité d'agir de l'UPSJ et, par conséquent, une option à éviter. Dans de telles circonstances, la voie pénale pourrait malheureusement de nouveau être retenue comme la solution de dernier recours.

\section{Références}

ABRAMSON, M. 1972. «The Criminalization of Mentally Disordered Behavior: Possible Side Effect of a New Mental Health Law » Hospital and Community Psychiatry 23 (4) : 101-105.

AdLer, F. 1986. "Jails as a Repository for Former Mental Patients " International Journal of Offender Therapy and Comparative Criminology 30 (3) : 225-236. 
BITTNER, E. 1967. « Police Discretion in Emergency Apprehension of Mentally Ill Persons » Social Problems 14 (3) : 278-292.

BonOvitZ, J.C. et BonOVITZ, J.S. 1981. « Diversion of the Mentally Ill into the Criminal Justice System: The Police Intervention Perspective » American Journal of Psychiatry 138 (7) : 973-976.

Brinded, P.M.J., Grant, F.E. et SMith, J.E. 1995. «The Spectre of Criminalization: Remand Admissions to the Forensic Psychiatric Institute, British Columbia, 1975-1990» Medecine, Science, and the Law 35 (4) : 59-64.

Brinded, P., Malcolm, F., Fairley, N. et Doyle, B. 1996. «Diversion versus Liaison: Psychiatric Services to the Courts, Wellington, New Zealand» Criminal Behaviour and Mental Health 6 : 167-176.

Brouillette, M.-J. et PARIS, J. 1991. « The Dangerousness Criterion for Civil Commitment: The Problem and a Possible Solution " Canadian Journal of Psychiatry 36 (4) : 285-289.

BRown, P. 1989. «Psychiatric Dirty Work Revisited: Conflicts in Servicing Nonpsychiatric Agencies " Journal of Contemporary Ethnography 18 (2): 182-201.

CASTEL, R. 1981. La gestion des risques: de l'antipsychiatrie à l'après psychanalyse, Paris : Éditions de Minuit.

ClSC DES FAubOuRgs 1996. Une alternative à l'exclusion, la judiciarisation et à l'incarcération. Les services d'urgence psychosociale, Montréal: CLSC des Faubourgs.

COHEN, N.L. et TSEMBeRIS, S. 1991. «Emergency Psychiatric Intervention on the Street » New Directions for Mental Health Services 52 : 3-16.

Comité de la SAnTÉ Mentale 1997. Défis. De la reconfiguration des services de santé mentale. Pour une réponse efficace et efficiente aux besoins des personnes atteintes de troubles mentaux graves (Rapport soumis au ministre de la Santé et des Services sociaux), Québec : Gouvernement du Québec.

Comité du BILAN D'IMPLANTATION DE LA PolitiQue DE SANTÉ MENTALE 1997. Ministère de la Santé et des Services sociaux. Orientations pour la transformation des services de santé mentale. Document de consultation, Québec : Gouvernement du Québec.

COMMISSION DE RÉFORME DU DROIT DU CANADA 1976. Désordre mental dans le processus pénal, Ottawa : Ministère des Approvisionnements et Services du Canada.

COMMISSION DE RÉFORME DU DROIT DU CANADA 1975. Processus pénal et désordre mental. Document de travail no 14, Ottawa : Imprimerie de la Reine.

DAVIS, S. 1992. "Assessing the "Criminalization" of the Mentally Ill in Canada » Canadian Journal of Psychiatry 37 (8) : 532-538.

Dear, M.J. et Wolch, J.R. 1987. Landscapes of Despair, Princeton, N.J. : Princeton University Press.

DORVIL, H. 1988. «L'accueil différentiel de la communauté à l'égard du malade mental » Santé mentale au Québec 13 (1) : 105-118.

FENNELL, P. 1991. «Diversion of Mentally Disordered Offenders from Custody » Criminal Law Review: 333-347. 
FinN, P. et Sullivan, M. 1989. « Police Handling of the Mentally Ill: Sharing Responsibility with the Mental Health System » Journal of Criminal Justice 17 (1) : $1-14$.

Foucault, M. 1961. Histoire de la folie, Paris : Plon.

FreEMAN, R.J. et RøesCH, R. 1989. « Mental Disorder and the Criminal Justice System: A Review » International Journal of Law and Psychiatry 12 (2-3) : 105-115.

GOFFMAN, E. 1968. Asiles. Études sur la condition sociale des malades mentaux, Paris : Les Éditions de Minuit.

GORDON, R.M. 1993. « Out to Pasture: A Case for the Retirement of Canadian Mental Health Legislation » Canadian Journal of Community Mental Health 12 (1) : 37-55.

Gordon, R.M. et Verdun-Jones, S.N. 1986. « Mental Health Law and Law Reform in the Commonwealth: The Rise of the "New Legalism" ? », Pp. 182 in Law and Mental Health. International Perspectives. vol.2, sous la direction de D.A. Weisstub. Toronto : Pergamon Press.

GreEN, T.M. 1997. « Police as Frontline Mental Health Workers: The Decision to Arrest or Refer to Mental Health Agencies » International Journal of Law and Psychiatry 20 (4) : 469-486.

Groupe DE TRAVAIL INTERMINISTÉRIEL M.S.S.S - M.J.Q. - M.S.G.Q. 1986. Plan d'action relatif aux services requis par la clientèle présentant le double problème de la maladie mentale et de la délinquance, Québec : Ministère de la Santé et des Services sociaux, Ministère de la Justice, Ministère du Solliciteur général.

HidAY, V.A. 1988. "Civil Commitment: A Review of Empirical Research » Behavioral Sciences and the Law 6 (1) : 15-43.

Hochstedler Steury, E. 1991. "Specifying "Criminalization" of the Mentally Disordered Misdemeanant " Journal of Criminal Law and Criminology $82(2)$ : 334-359.

Hodgins, S. et CôTÉ, G. 1990. «Prévalence des troubles mentaux chez les détenus des pénitenciers du Québec » Santé mentale au Canada 38 (1) : 1-5.

Holley, H.L. et ARboledA-FloreZ, J. 1988. « Criminalization of the Mentally Ill: Part 1. Police Perception » Canadian Journal of Psychiatry 33 : 81-86

HolsteIn, J.A. 1993. Court-Ordered Insanity. Interpretive Practice and Involuntary Commitment, New York : Aldine de Gruyter.

James, D.V. et Hamilton, L.W. 1991. "The Clerkenwell Scheme: Assessing Efficacy and Cost of a Psychiatric Liaison Service to a Magistrates' Court » BMJ. British Medical Journal 303 (août) : 282-285.

James, D.V. et Hamilton, L.W. 1992. « Setting Up Psychiatric Liaison Schemes to Magistrates'Courts: Problems and Practicalities » Medicine, Science, and the Law 32 (2) : 167-176.

Joseph, P.A. et PotTer, M. 1993a. "Diversion from Custody. I: Psychiatric Assessment at the Magistrates' Court » British Journal of Psychiatry 162 : 325-330.

Joseph, P.A. et PotTer, M. 1993b. « Diversion from Custody. II: Effect on Hospital and Prison Resources » British Journal of Psychiatry 162 : 330-334. 
Laberge, D., Landreville, P., Morin, D., Robert, M. et Soullière, N. 1991. Le traitement judiciaire des personnes connaissant des problèmes de santé mentale (Cahier no 13), Montréal : Les cahiers du GRAPPP.

Laberge, D., Landreville, P., Morin, D., Robert, M. et Soullière, N. 1995. Maladie mentale et délinquance: deux figures de la déviance devant la justice pénale, Collection Perspectives criminologiques, Montréal: Les Presses de l'Université de Montréal.

LABERGE, D. et MORIN, D. 1992. Les clientèles " psychiatrie-justice» : problèmes de prise en charge et d'intervention, Montréal : Les cahiers du GRAPPP.

LABERGE, D. et MORIN, D. 1995. "The Overuse of Criminal Justice Dispositions. Failure of Diversionary Policies in the Management of Mental Health Problems » International Journal of Law and Psychiatry 18 (4) : 389-414.

LABERGe, D., MOrin, D. et Robert, M. 1996. Criminalisation et maladie mentale présumée : les réponses du système judiciaire, Montréal : Les cahiers du GRAPPP.

LAMB, R.H. et GRANT, R.W. 1982. « The Mentally Ill in an Urban County Jail » Archives of General Psychiatry 39 (Janvier) : 17-22.

LAMB, R. et WeinBerger, L.E. 1998. «Persons with Severe Mental Illness in Jails and Prisons: A Review » Psychiatric Services 49 (4) : 483-492.

Landreville, P., Blankewoort, V. et Pires, A. 1981. Les coûts sociaux du système pénal, Montréal : École de criminologie, Université de Montréal.

Lefebvre, Y. 1985. Psycauses, Montréal : Recherche subventionnée par Santé et Bien-être social Canada.

LOVELL, A.M. 1996. « Cœrcion and Social Control: A Framework for Research on Aggressive Strategies in Community Mental Health », Pp. 148-166 in Coercion and Aggressive Community Treatment. A New Frontier in Mental Health Law, sous la direction de D.L. Dennis et J. Monahan. New York : Plenum Press.

MarchetTI, A.-M. 1996. "Pauvreté et trajectoire carcérale », Pp. 177-197 in Approches de la prison, Perspectives criminologiques, sous la direction de C. Faugeron et A.C.P. Chauvenet. Bruxelles : De Bœck.

Migneault, P. et O’Neil, J. 1988. Consentement éclairé et capacité en psychiatrie. Aspects cliniques et juridiques, Verdun : Éditions Douglas.

PATCH, P.C. et ARrigo, B.A. 1999. "Police Officer Attitudes and Use of Discretion in Situations Involving the Mentally Ill: The Need to Narrow the Focus » International Journal of Law and Psychiatry 22 (1) : 23-35.

Peyrot, M. 1982. "Caseload Management: Choosing Suitable Clients in a Community Health Clinic Agency » Social Problems 30 : 157-167.

PORPORINO, F.J. et MOTIUK, L.L. 1993. «La libération conditionnelle des délinquants atteints de troubles mentaux » Forum 5 (3) : 19-22.

RÉGIE RÉGIONALE DE LA SANTÉ ET DES SERVICES SOCIAUX DE MONTRÉALCENTRE 1994a. Groupe de travail clientèles santé mentale-justice dirigé par D. Marineau. Scénario de développement Service d'urgence psychosociale, Montréal : RRSSS.

RÉGIE RÉGIONALE DE LA SANTÉ ET DES SERVICES SOCIAUX DE MONTRÉALCENTRE 1994b. Groupe de travail multisectoriel sour la coordination du 
Secteur des services de santé mentale de la RRSSS de M-C dirigé par D. Marineau. Services d'urgence psychosociale. Clientèle santé mentale-justice. Concept de services, Montréal : RRSSS.

RÉGIE RÉGIONALE DE LA SANTÉ ET DES SERVICES SOCIAUX DE MONTRÉALCENTRE 1996. Services d'urgence psychosociale. Clientèle santé mentale-justice. Plan de démarrage, Montréal : RRSSS.

Robertson, G.B. 1987. Mental Disability and the Law in Canada, Toronto: Carswell.

SCHEFf, T.J. 1966. Being Mentally Ill. A Sociological Theory, Chicago : Aldine Publishing.

SHABO, H.E. 1994. «Deinstitutionalization, Homelessness and the Criminalization of the Mentally Ill in the United States of America » International Medical Journal 1 (1) : 2-4.

TABle de CONCERTATION PSyChiatrie-JUSTICE DE Montréal 1989. Plan d'organisation des services de santé mentale dans le secteur psychiatrie-justice pour la région 06- $A$. Bilan et recommandations soumis aux CRSSSMM. Document de travail, Montréal.

TePlin, L.A. 1983. «The Criminalization of the Mentally Ill: Speculation in Search of Data » Psychological Bulletin 94 (1) : 54-67.

TEPLIN, L.A. 1984. « Managing Disorder. Police Handling of the Mentally Ill », Pp. 157-175 in Mental Health and Criminal Justice, sous la direction de L.A. Teplin. Beverly Hills : Sage.

Teplin, L.A. et PruetT, N.S. 1992. "Police as Streetcorner Psychiatrist: Managing the Mentally Ill » International Journal of Law and Psychiatry 15 (2) : 139-156.

Wachholz, S. et Mullaly, R. 1993. «Policing the Deinstitutionalized Mentally Ill: Toward an Understanding of Its Function " Crime, Law and Social Change 19 (3) : 281-300.

WARren, C.A.B. 1981. "New Forms of Social Control: The Myth of Deinstitutionalization » American Behavioral Scientist 24 (6) : 724-740.

Way, B.B., Evan, M.E. et BANKS, S.M. 1993. « An Analysis of Police Referrals of 10 Psychiatric Emergency Rooms » Bulletin of the American Academy of Psychiatry and the Law 21 (4) : 389-397. 